(C) 2014 IEEE. Personal use of this material is permitted. Permission from IEEE must be obtained for all other uses, in any current or future media, including reprinting/republishing this material for advertising or promotional purposes, creating new collective works, for resale or redistribution to servers or lists, or reuse of any copyrighted component of this work in other works. 


\title{
Circularly-Polarized Conical-Beam Antenna with Wide Bandwidth and Low Profile
}

\author{
Wei Lin, Student Member, IEEE and Hang Wong, Senior Member, IEEE
}

\begin{abstract}
This work introduces a circularly-polarized (CP) conical-beam antenna with low profile and wide impedance \& axial ratio (AR) bandwidths. The antenna structure simply consists of a wideband monopolar patch antenna and eight parasitic loop elements for generating vertically polarized electric field $E_{\theta}$ and horizontally polarized field $E_{\varphi}$, respectively. The proposed antenna is low profile but wideband because of merging the $T M_{01}$ and $T M_{02}$ modes at around the center frequency. In order to obtain the wideband $C P$ radiation, the eight parasitic loop stubs are printed and placed sequentially along the radiating edge of the patch. With the resultant radiations from both the patch antenna and the loop stubs, the $C P$ conical-beam radiation pattern can be obtained. In addition, a parametric study of the shorting vias effect, number of loop elements, and loop widths are demonstrated how to design this wideband $C P$ antenna. The antenna exhibits the wide impedance bandwidth of $28 \%$ from 4.9

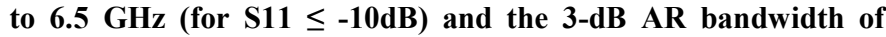
$14.4 \%$ from 5.8 to $6.7 \mathrm{GHz}$. The thickness of this antenna is only $0.06 \lambda$ in terms of free-space wavelength at its lowest operation frequency. Stable omni-directional conica-beam radiation patterns are observed over the operating bandwidth with the peak gain of $4.9 \mathrm{dBic}$. The antenna can cover the uplink of standard $\mathrm{C}$ band $(5.85$ to $6.425 \mathrm{GHz})$ for satellite communications.
\end{abstract}

Index Terms-Circular polarization, conical-beam, wide bandwidth, low profile, patch antenna.

\section{INTRODUCTION}

C IRCULARLY polarized (CP) antennas with conical beam have drawn more and more attention with the rapid development of the wireless communications in recent years. $\mathrm{CP}$ antennas with conical-beam radiation patterns are desirable to be mounted on moving vehicles for communicating with geostationary satellites due to the freedom of orientation in satellite tracking.

Many efforts have been demonstrated in $\mathrm{CP}$ conical-beam antenna designs [1-16]. For example, a $\mathrm{TM}_{21}$ mode patch antenna [1] excited by multiple feedings can generate the CP conical-beam radiation. This antenna has a simple structure and yields an AR bandwidth of $4 \%$. In addition to the patch antenna, dipole antennas could also generate the $\mathrm{CP}$ conical-beam radiation patterns. One of the instances reported in [3], two orthogonal dipoles with a $90^{\circ}$ phase difference and with a chosen proper distance to the ground reflector can transform the radiation pattern from the broadside direction to the conical beam. The CP dipoles obtain the AR bandwidth of 3\% but the antenna results in a thicker structure than that of the candidate in [1]. Moreover, four inclined monopoles [4] with a feeding network are introduced to achieve the CP conical-beam radiation pattern. The profile is also high and the reported AR bandwidth is only $2.8 \%$. Furthermore, a circular waveguide [5] with the excitations of $\mathrm{TE}_{01}$ and $\mathrm{TM}_{01}$ modes achieves the $\mathrm{CP}$ conical-beam radiation pattern with the AR bandwidth of $4.8 \%$. Admittedly, someone demonstrated a loop antenna [7] could produce the conical-beam radiation in a very low-profile structure $(0.064 \lambda)$ but retain the narrow AR bandwidth of $0.5 \%$ only. In addition, the use of eight-branch folded loop stubs was tried to generate $\mathrm{CP}$ radiation with conical beam [8-9]. However, the $\mathrm{CP}$ characteristics are quite poor (the impedance bandwidth is less than $2 \%$ and the AR bandwidth is not presented) although it has a low profile $(0.07 \lambda)$. Moreover, other reported designs in [10-13] can also generate CP conical-beam radiations. However, their AR bandwidths are still very narrow (less than $0.5 \%$ ). All above examples, exhibit that the patch, the dipole, the monopole, the waveguide, and the loop antennas could generate the $\mathrm{CP}$ conical-beam radiation pattern; nonetheless, it is very challenging to have the wide AR bandwidth in the conical-beam antennas. Although a two-arm spiral antenna excited in phase can obtain a wide AR bandwidth from 3.3 to $9.6 \mathrm{GHz}$, it has a directional radiation pattern in the azimuth plane and a large size [14]. In addition, some proposed a dielectric bird-nest antenna [15] and an L-shaped probe fed high-order mode patch antenna [16] can deliver the wide AR bandwidths up to $54.9 \%$ [15], however, their antenna geometries are complex that causes harshness in fabrication.

In this paper, we propose a new $\mathrm{CP}$ conical-beam antenna which is low profile, simple, and wideband. We have successfully employed two new techniques of utilizing loop stubs for achieving wideband $\mathrm{CP}$ conical-beam radiation: Coupling excitation and Shorting vias at the ends of the loop stubs. Such that we can combine a monopolar patch and eight parasitic loop stubs to obtain two orthogonally-polarized electric fields with the excitation of $90^{\circ}$ phase difference at the same time for generating the wideband CP radiation with conical-beam pattern. In addition, the antenna thickness is only $0.06 \lambda$ in terms of free-space wavelength at its lowest operation frequency. Moreover, it has a simple structure that can be fabricated by the printed-circuit-board (PCB) technology.

Antenna design and operation principle are reported in the following Part II. And several important design considerations are discussed in Part III. Measured results are presented in Part IV. Finally Part V is the conclusion. 


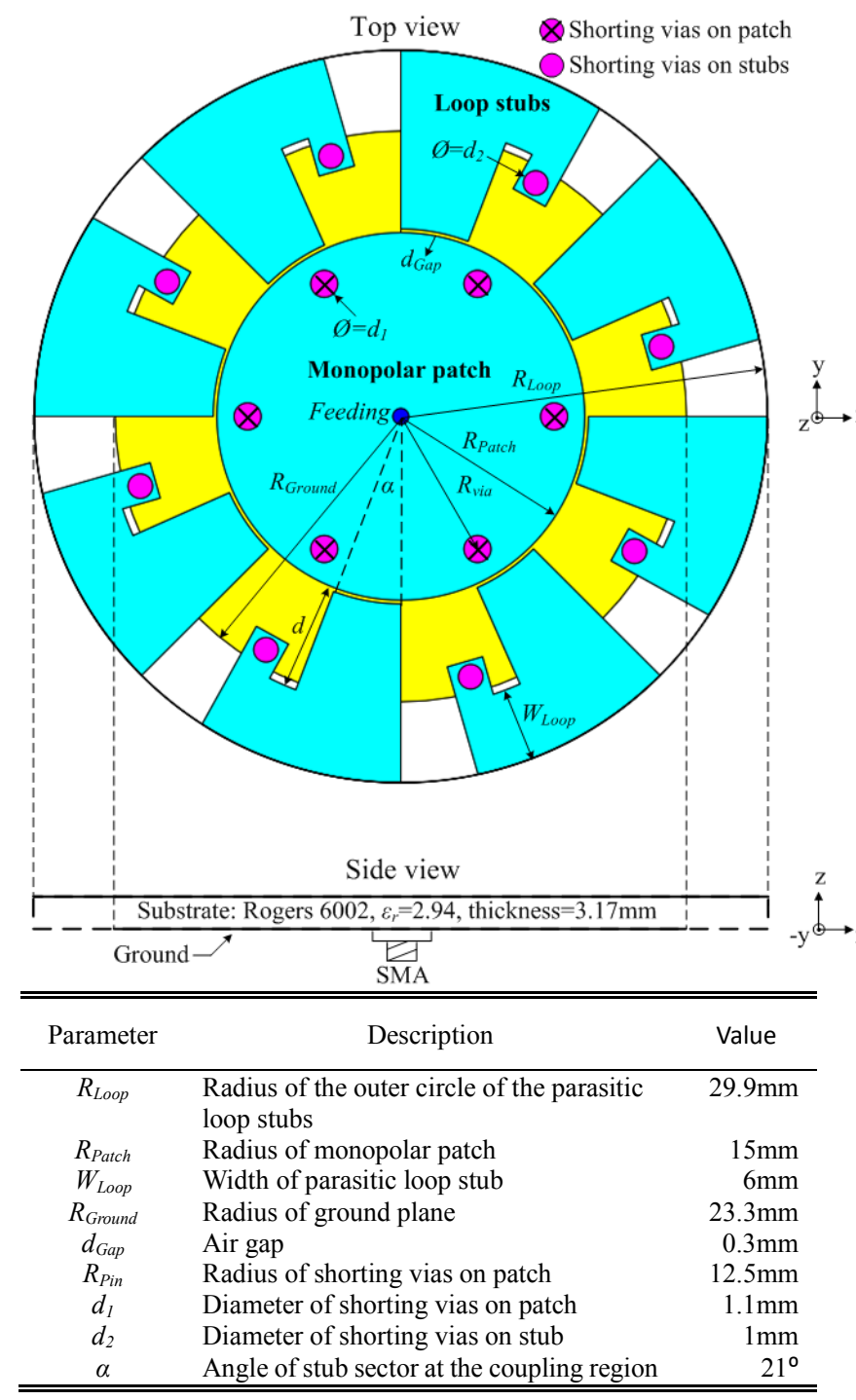

Fig. 1. Configuration of the printed wideband CP conical-beam patch antenna.

\section{Antenna Design}

\section{A. Antenna Configuration}

The proposed antenna consists of two sets of radiating elements. The primary radiator is a center-fed wideband monopolar patch antenna with six vertical shorting vias connected to the ground. This monopolar patch antenna element is modified from our prior work in [10]. As shown in Fig.1, the monopolar patch has a circular shape and is placed at the center of the antenna excited by a coaxial feed. Another set of radiating elements is the eight parasitic loop stubs which are located sequentially along the edge of the patch. One end of each stub is connected to the ground by an additional shorting via. The other end of the stub is open-circuited for coupling the energy from the patch. Since the stub is connected to the ground plane, this manner forms a loop-stub structure. As the result, the proposed antenna has two radiations: vertically polarized radiation from the monopolar patch and horizontally polarized radiation from the coupling loop stubs respectively. Their resultant radiations contribute a $\mathrm{CP}$ conical-beam radiation

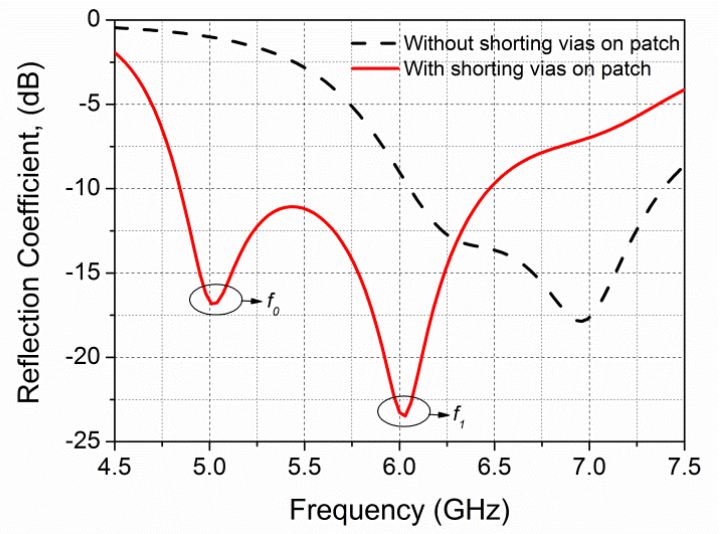

Fig. 2. Reflection coefficients with and without the shorting vias on the patch.

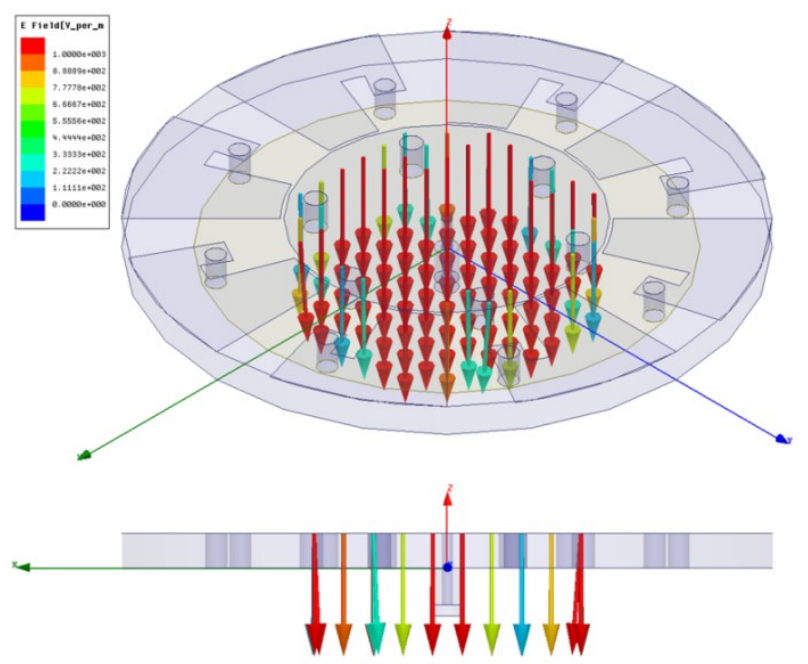

(a)

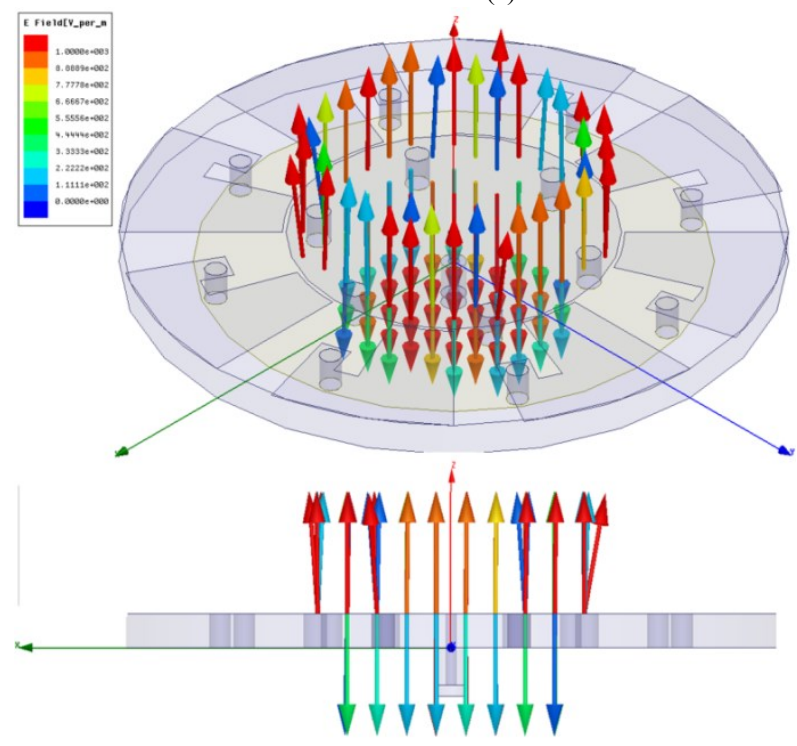

(b)

Fig. 3. Electric field distributions on the monopolar patch: (a) at $f_{0}(5 \mathrm{GHz})$ and (b) at $f_{l}(6 \mathrm{GHz})$.

with wideband characteristics. To provide more design descriptions, we are going to give more explanations in the design of the monopolar patch and the coupling loop stubs in the following part. And the detailed dimensions of the parameters are listed in the table of Fig.1. 


\section{B. Primary radiator: Wideband Monopolar Patch}

The center-fed monopolar patch antenna with conductive shorting vias has the characteristics of wide impedance bandwidth, very low profile and stable vertically-polarized conical-beam radiation patterns across the operating bandwidth [17]. It utilizes six shorting vias to control the resonant frequencies of $\mathrm{TM}_{01}$ and $\mathrm{TM}_{02}$ modes working closer to the center frequency through adjusting the location of all vias defined by $R_{v i a}$ and the diameter of the via $d_{l}$, such that the wide impedance bandwidth can be obtained. The comparison of the reflection coefficients between antennas with and without shorting vias on the monopolar patch is shown in Fig. 2. It is seen that the presence of the shorting vias increases the impedance bandwidth from $19.9 \%$ to $29.1 \%$, and shifts the operation frequency to the lower frequency which implies the effect of miniaturization. Electric field distributions on the monopolar patch of the proposed antenna at the two resonant frequencies $f_{0}(5 \mathrm{GHz})$ and $f_{l}(6 \mathrm{GHz})$ are shown in Fig. 3. It is seen clearly that $\mathrm{TM}_{01}$ mode is excited at $f_{0}$ and $\mathrm{TM}_{02}$ mode is excited at $f_{l}$. As the result, wide impedance bandwidth has been achieved since the two modes move closer by the introduction of the six shorting vias on patch. The design frequency of this antenna can be determined by the radius of the monopolar patch $R_{\text {Patch }}$ which is calculated by the following equations [17]:

$$
\begin{gathered}
k_{0} \sqrt{\varepsilon_{r}} R_{\text {eff }}=3.83171 \\
R_{\text {eff }}=R_{\text {Patch }} \sqrt{1+\frac{2 t}{\pi R_{\text {Patch }} \varepsilon_{r}}\left(\operatorname{In} \frac{\pi R_{\text {Patch }}}{2 t}+1.7726\right)}
\end{gathered}
$$

where $k_{0}$ is the wavenumber of the $\mathrm{TM}_{02}$ mode in free space, $R_{\text {eff }}$ is the effective radius for considering the fringing fields and $t$ is the thickness of the substrate.

\section{Parasitic Radiator: Coupling Loop Stubs}

Since the wideband monopolar patch antenna has the stable conical-beam radiation patterns with vertically-polarized electric field $E_{\theta}$ across the entire bandwidth. If there is any horizontally-polarized electric field $E_{\varphi}$ generated with $90^{\circ}$ phase difference to the electric field $E_{\theta}$ with similar conical-beam pattern, then the resultant field from both $E_{\theta}$ and $E_{\varphi}$ could generate the CP wave propagation. To realize this, coupling loop stubs with end shorting vias are introduced as parasitic radiator to generate $E_{\varphi}$ with conical-beam pattern.

$\mathrm{CP}$ radiation requires that the amplitudes of $E_{\theta}$ and $E_{\varphi}$ are equal and their phase difference is $90^{\circ}$. We adopt eight coupling loop stubs with end shorting vias placed along the circular patch edge sequentially. The antenna with this manner can generate horizontally-polarized electric field $E_{\varphi}$. Since the stubs are formed in round shape and connected to the ground, they would create an enclosed loop current (as in Fig. 12) which contributes the radiation of horizontally-polarized electric field $E_{\varphi}$. As shown in Fig. 4, both the monopolar patch and the coupling loop stubs provide conical-beam radiation patterns in the vertical plane. The monopolar patch generates the vertically-polarized electric field $E_{\theta}$ while the loop stubs are the

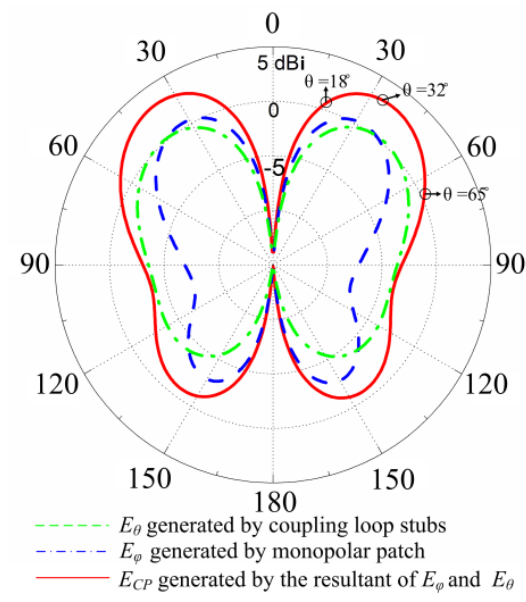

Fig. 4. Electric field components of the $\mathrm{CP}$ radiation at $6 \mathrm{GHz}\left(\varphi=0^{\circ}\right)$.

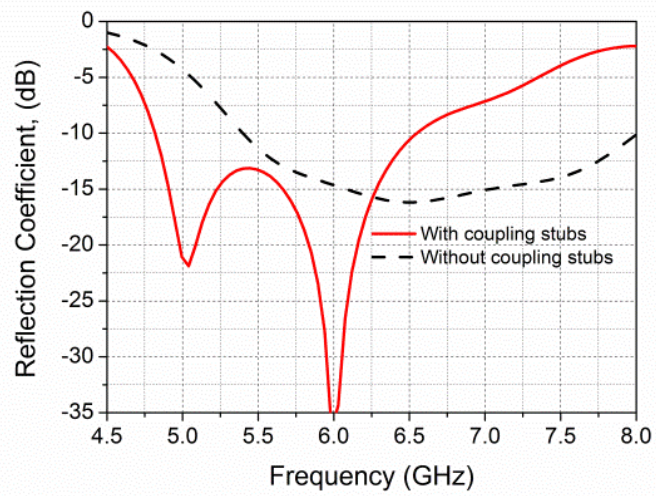

Fig. 5. Reflection coefficient comparison between the monopolar patch with and without the coupling stubs.

horizontally-polarized electric field $E_{\varphi}$. The $90^{\circ}$ phase difference between the two radiations is realized by making the total length of each stub around half wavelength $\lambda_{g} / 2$ at the center frequency in the initial design. There is a distance $d$ between the patch edge and the round-shaped loop as in Fig. 1. The loop stub at the coupling region with the same length $d$ can be regarded as a section of transmission line on which traveling wave flows. Therefore, to control the proper phase difference with $90^{\circ}$, the distance $d$ should satisfy the equations below:

$$
\begin{gathered}
W_{\text {Loop }}+d=R_{\text {Loop }}-R_{\text {Patch }} \\
d=\lambda /\left(4 * \sqrt{\varepsilon_{e}}\right)
\end{gathered}
$$

In which $\lambda$ is the wavelength in free space and $\varepsilon_{e}$ is the effective permittivity.

The coupling stubs will introduce the loading effect for the monopolar patch, such that the operating frequency will move to the lower band as seen in Fig. 5. Since the impedance bandwidth will be affected by the effective thickness of the substrate, the impedance bandwidth becomes a bit narrower but is still wide enough to cover the entire AR bandwidth as shown in Fig. 6. Axial ratio beamwidth and axial ratio bandwidth are two key parameters to evaluate $\mathrm{CP}$ characteristics for conical-beam radiation. Fig. 7 shows the AR values at the same vertical plane $\left(\varphi=0^{\circ}\right)$ as radiation patterns plotted in Fig. 4. It is seen that the $3 \mathrm{~dB}$ AR beamwidth is very wide (up to elevation 


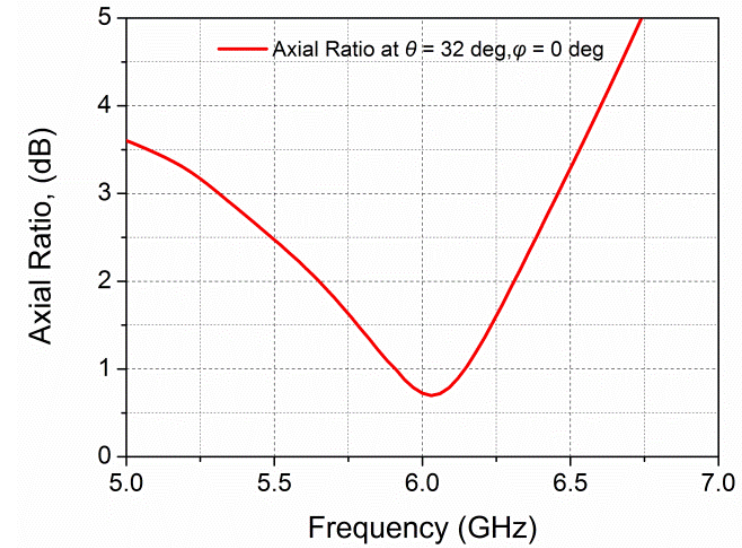

Fig. 6. Axial ratio bandwidth at the vertical plane $\left(\theta=32^{\circ}, \varphi=0^{\circ}\right)$.

angle $\theta=54^{\circ}$ ) and the best $\mathrm{CP}$ radiation appears at $\theta=32^{\circ}$. In addition, the $3 \mathrm{~dB}$ RHCP gain beamwidth is also indicated in Fig. 4 which is from $\theta=18^{0}$ to $\theta=65^{\circ}$. Therefore, the overlapped beamwidth can cover a wide angle range which is from $\theta=18^{0}$ to $\theta=54^{0}$.

Another parameter is the AR bandwidth, which is defined for a certain elevation angle $\theta$ at vertical plane. Fig. 6 shows the AR bandwidth at $\theta=32^{\circ}\left(\varphi=0^{\circ}\right)$ where the best CP radiation appears (highest gain and lowest AR). It can be seen that the wide AR bandwidth from 5.34 to $6.45 \mathrm{GHz}(18.8 \%)$ is obtained. In addition, for our targeted application band: uplink of standard C band (5.8 to $6.425 \mathrm{GHz}$ ) for geostationary satellite communication, the AR beamwidth of the proposed antenna is also given in Fig. 8. The overlapped $3 \mathrm{~dB}$ AR beamwidth is wide which is from $27^{\circ}$ to $51^{\circ}$ in the vertical plane.

\section{PARAMETRIC STUDY}

For achieving the wideband $\mathrm{CP}$ radiation, some design considerations are very important. To exhibit a full picture and the understanding of this design, a parametric study is carried out in this part. There are three design concerns including: (a) coupling excitation for the loop stubs; (b) presence of the shorting vias at the end of stubs; (c) stub width and number of the loop stubs; (d) effect of the ground size $R_{\text {Ground }}$ and (e) polarizations.

\section{(a) Coupling excitation for the loop stubs}

The coupling excitation for the loop stubs is critical because it will affect the electric field mode inside the circular cavity. If the loop stubs are connected directly with the monopolar patch, the impedance matching will deteriorate totally as shown in Fig. 9. However, when a gap $d_{\text {Gap }}$ is provided in between the patch and the stubs, the wideband characteristic of the patch can be maintained. This gap also introduces a good environment for coupling excitation to the loop stubs. The effects of the different air gap for impedance and AR bandwidths are plotted. It is observed that the wide impedance matching is obtained with the present of the air gap. Reflection coefficient does not change much with the variation of gap $d_{\text {Gap }}$ from $0.1 \mathrm{~mm}$ to $0.9 \mathrm{~mm}$ (all other parameters are the same as in Fig. 1).

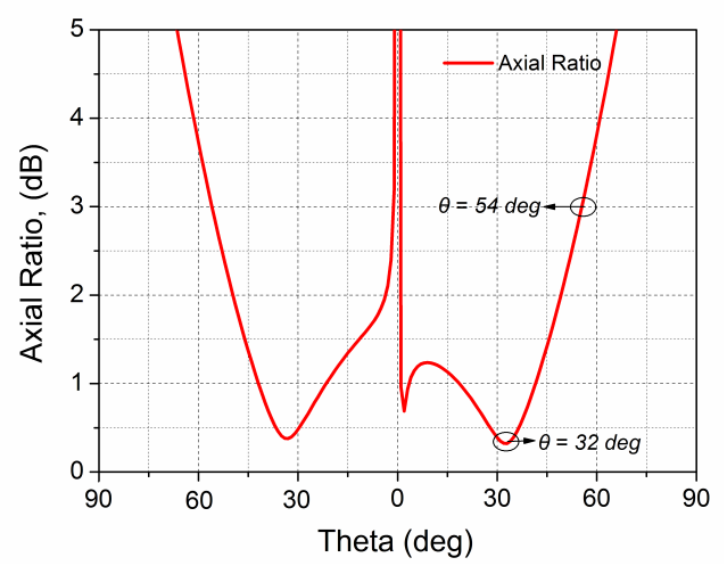

Fig. 7. Axial Ratio value of $\varphi=0^{\circ}$ plane at $6 \mathrm{GHz}$.

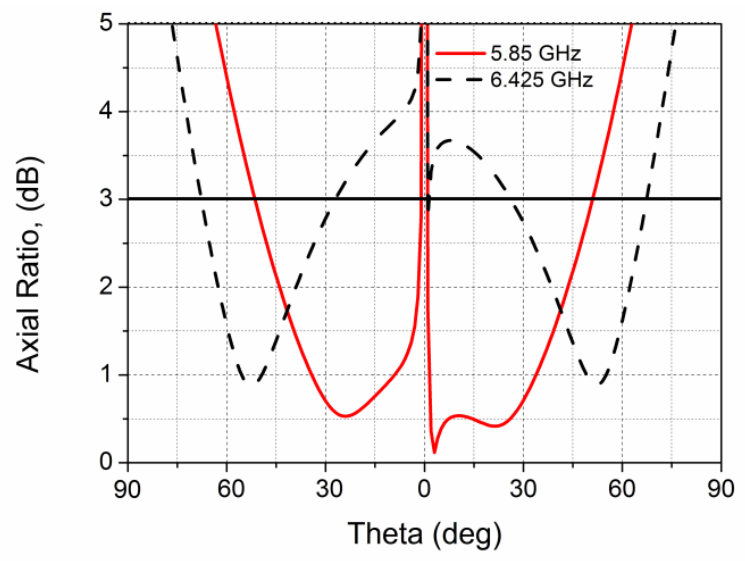

Fig. 8. Axial ratio beamwidth $\left(\varphi=0^{\circ}\right)$ at the two edge frequencies of uplink of standard $\mathrm{C}$ band.

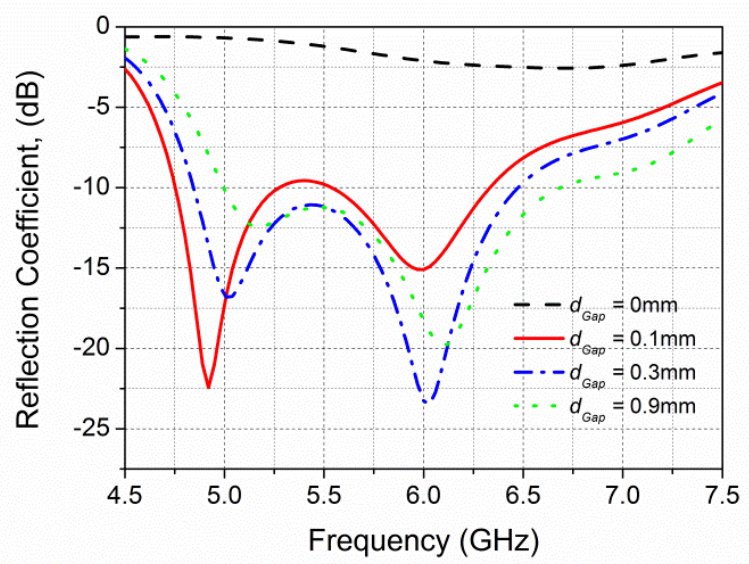

Fig. 9. Reflection coefficients with the different air gap $d_{G a p}$.

Nevertheless, the axial ratio bandwidth changes according to $d_{\text {Gap }}$ as in Fig. 10 and there is an optimal value for $d_{\text {Gap }}$. In order to obtain the maximum AR bandwidth $d_{G a p}=0.3 \mathrm{~mm}$ is chosen for the final design.

\section{(b) Shorting vias at the end of stubs}

Shorting vias at the end of coupling loop stubs are also crucial for obtaining wideband $\mathrm{CP}$ radiation. Fig. 11 shows the comparison of AR bandwidths and reflection coefficients with and without adding the shorting vias at the end of loop stubs. Apparently there is poor $\mathrm{CP}$ radiation when those shorting vias 


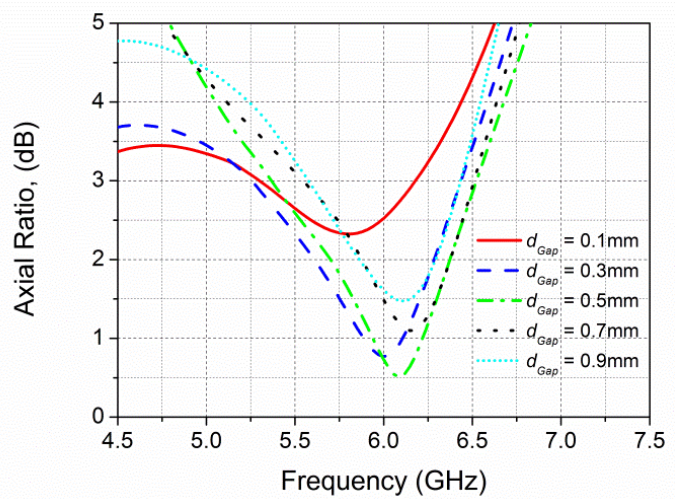

Fig. 10. Axial ratio bandwidth at the vertical plane $\left(\theta=32^{\circ}, \varphi=0^{\circ}\right)$ with the different air gap $d_{\text {Gap. }}$

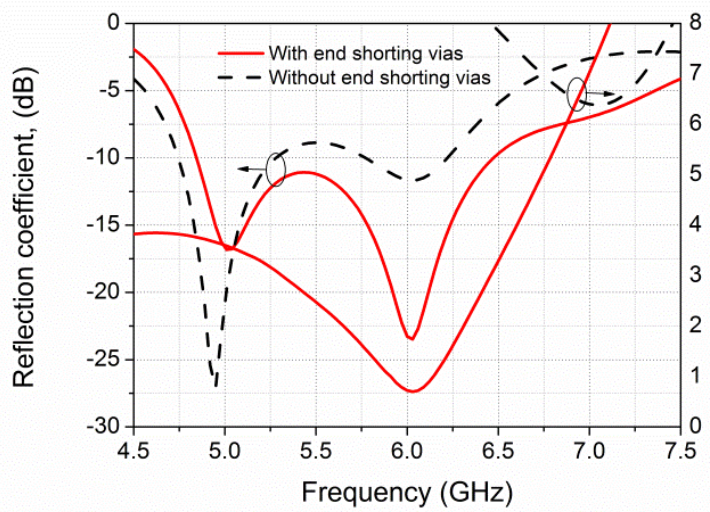

$\frac{0}{0}$
$\frac{0}{0}$
$\frac{0}{\sqrt[0]{0}}$
$\frac{x}{\frac{\pi}{0}}$
$\frac{\pi}{x}$

Fig. 11. Axial ratio bandwidths and reflection coefficients with and without the shorting vias on the loop stubs.

are removed. However, impedance matching does not change much due to the coupling excitation technique. In the proposed design, the end shorting vias are critical for wideband $\mathrm{CP}$ conical-beam radiation. This working principle can be explained by plotting the current distributions on the antenna at the center frequency of $6 \mathrm{GHz}$ as shown in Fig. 12. An in-phase current loop is required to generate horizontally-polarized radiation with conical-beam radiation pattern. It is seen obviously that the presence of shorting vias provide a regular in-phase current loop for every loop stub element. However, without end shorting vias, the current will be reflected by the stub end which destroys in-phase current loop along the circle. Therefore, the shorting vias can effectively prolong the current path to allow travelling wave flowing on each loop stub so that in-phase loop current will appear. Unlike antennas with the loop current to generate omni-directional pattern like magnetic dipole at the $\theta=90^{\circ}$ plane in [18-20], for this design, horizontally-polarized conical-beam pattern is achieved due to travelling wave on stubs and the ground plane below. The directions of the currents $J_{\text {patch }}$ and $J_{\text {stub }}$ are also indicated in the figure. Because $J_{\text {patch }}$ generated by the monopolar patch is in advance to $J_{\text {stub }}$ by the stubs, right-handed circular polarization (RHCP) is realized.

\section{(c) Stub width and number of the loop stubs}

The choices of the stub width and stub numbers are other important concerns for obtaining the wide AR bandwidth. Fig. 13 shows three antenna designs with different stub widths and

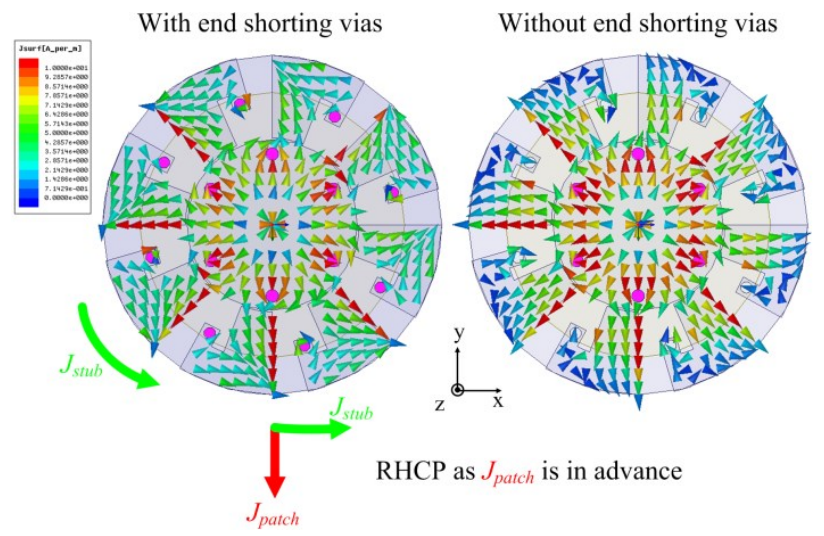

Fig. 12. Current distributions at $6 \mathrm{GHz}$ with and without the end shorting vias
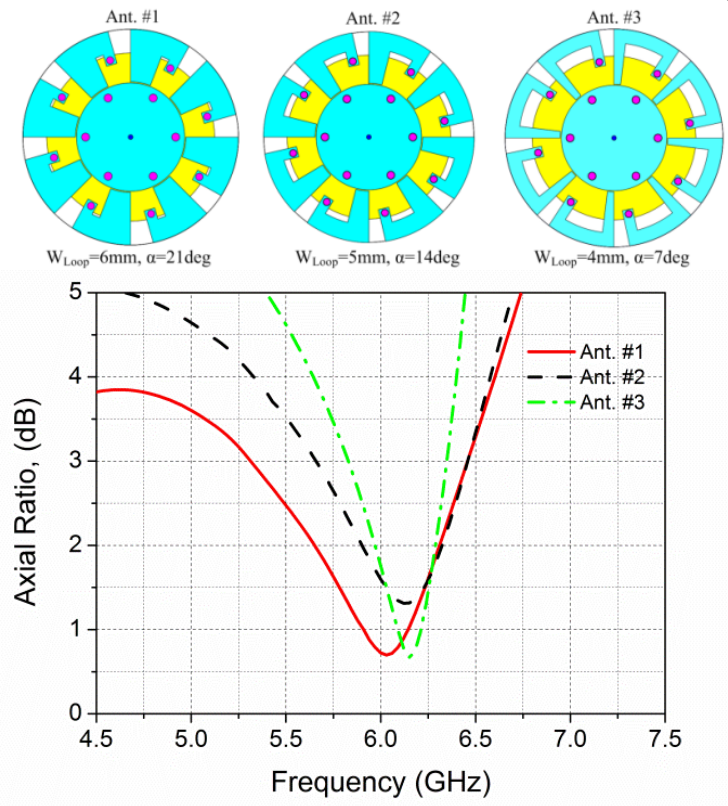

Fig. 13. Axial ratio bandwidths at the vertical plane with different stub widths.

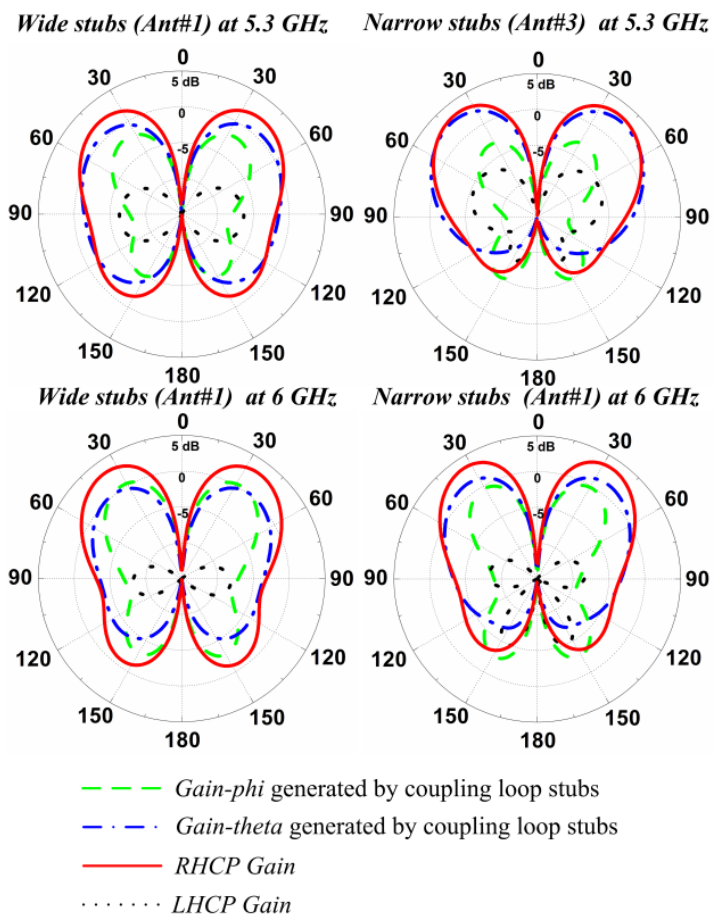

Fig. 14. Radiation patterns for antennas with the different stub widths 

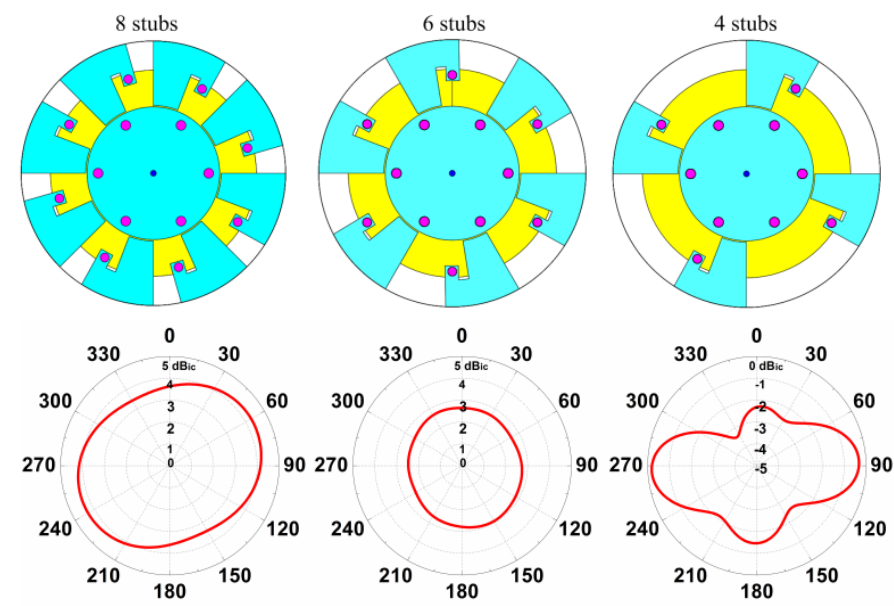

Fig. 15. Antenna with the different loop stub numbers and RHCP gain at the azimuth plane.

their axial ratio bandwidths. It can be concluded that a wider AR bandwidth can be achieved by a wider stub. For obtaining the widest AR bandwidth as our design target, stub parameters $W_{\text {Loop }}=6 \mathrm{~mm}, \alpha=21 \mathrm{deg}$ are chosen in the final design.

Wider stubs are required for obtaining the wideband $\mathrm{CP}$ radiation. This can be explained by reviewing the operating principle in Part II. Loop stubs as parasitic radiator needs to couple the energy from the primary radiator: monopolar patch. The width of stub will determine the amount of the coupled energy and the pattern variation of $E_{\varphi}$ generated from the loop stubs. As seen in Fig. 14, at $5.3 \mathrm{GHz}$ the magnitude difference between Gain-theta and Gain-phi at $\theta=32^{\circ}$ is $1.8 \mathrm{dBi}$ for the wide stubs but $5.1 \mathrm{dBi}$ for the narrow stubs. This means for the narrow stubs the coupled energy from primary radiator is not sufficient for generating $\mathrm{CP}$ radiation. It is also seen that from 5.3 to $6 \mathrm{GHz}$ the pattern variation of antenna with the wide loop stubs is small, so that wideband performance is achieved. However, the patterns of antenna with the narrow stubs change greatly thus resulting in narrowband effect.

Fig. 15 to Fig. 17 show the effect of the different stub numbers. It can be observed that both the antenna gain and the AR bandwidth drop when the number of stubs decreases. In addition, enough stub numbers are required for keeping the good omni-directional radiation characteristics. For example, $\mathrm{AR}$ value varies greatly at the azimuth plane if the number of stubs is four. Therefore, eight stubs are chosen at the end.

\section{(d) Effect of the ground size $R_{\text {Gound }}$}

Another important concern in this design is the ground size $R_{\text {Ground. }}$ The radius of the ground plane cannot cover the entire loop stubs as this manner will damage the operating modes of the monopolar patch as well as its conical-beam radiation pattern. Therefore, both the impedance matching and the $\mathrm{CP}$ radiation will be totally destroyed as shown in Fig. 18 and Fig. 19. The optimized size of ground should be slightly larger than the position of the end shorting vias of the loop stubs as seen in Fig. 1. Therefore, finally $R_{\text {Ground }}=23.3 \mathrm{~mm}$ is chosen for the widest impedance and AR bandwidths.

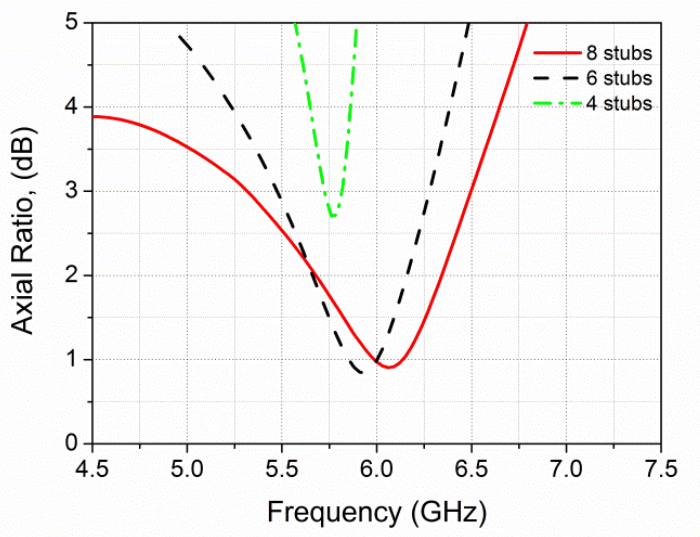

Fig. 16. Axial ratio bandwidth at the vertical plane with different stub numbers.

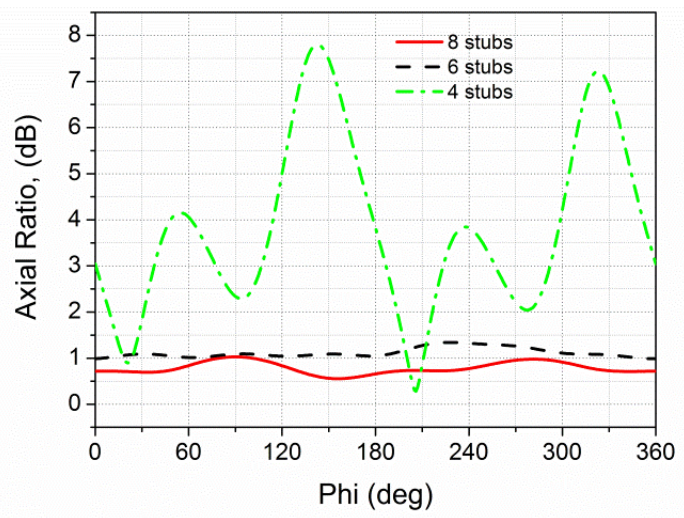

Fig. 17. AR variations at the azimuth plane with different stub numbers.

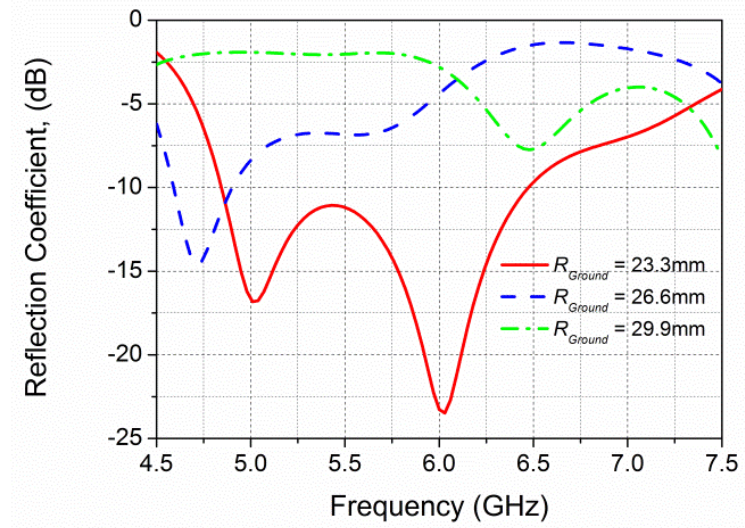

Fig. 18. Reflection coefficients of antenna with different ground size $R_{\text {Gound. }}$

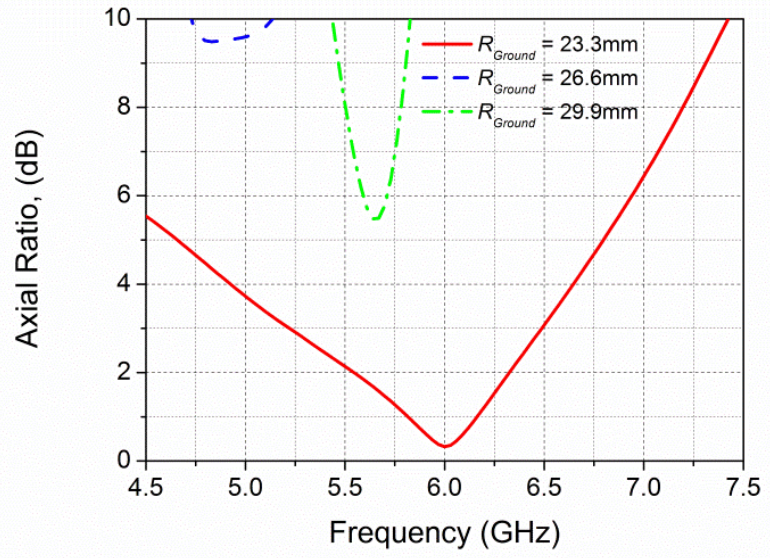

Fig. 19.Axial Ratio bandwidths of antenna with different ground size $R_{\text {Gound }}$ 


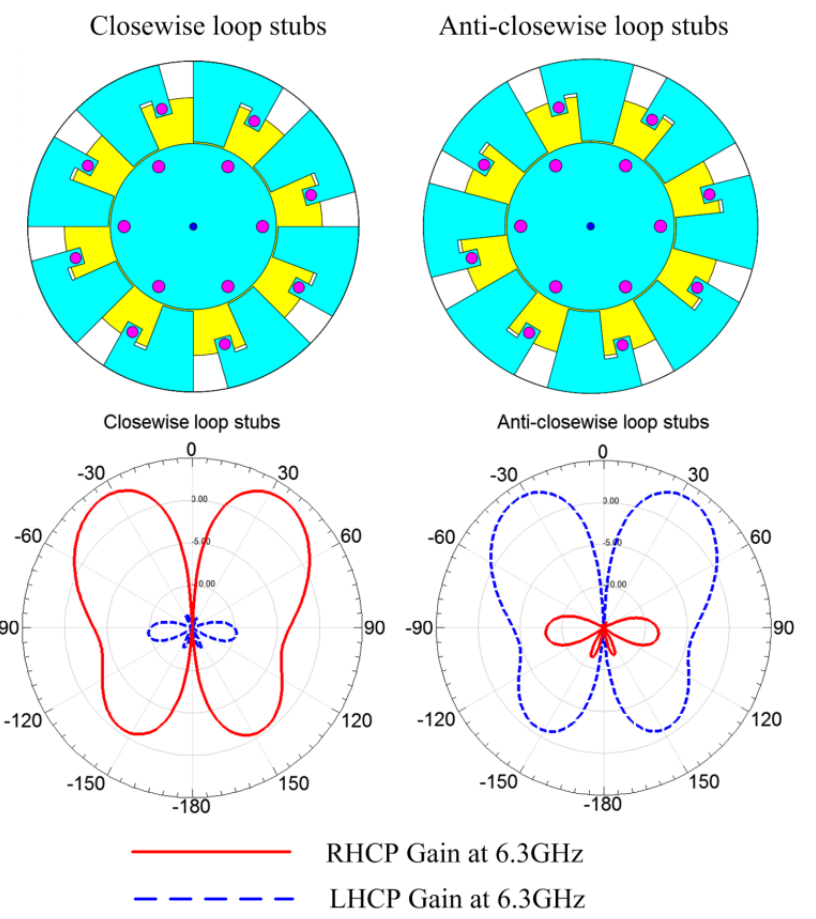

Fig. 20. Circular polarization types of different stub orientations.

\section{(e) Polarizations}

Right-handed circular polarization (RHCP) and left-handed circular polarization (LHCP) can be alternated by changing the orientation of the loop stubs. Fig. 20 shows the same antenna with the different stub orientations. The polarization can be reverted when the stub orientation is changed to the opposite.

\section{MEASURED Results}

The proposed antenna was fabricated as shown in Fig. 21. All the conductive vias are realized by the plate via holes. Due to the simple structure, it is low-cost and can be easily fabricated by PCB manufacture technology. All simulated results are from Ansoft HFSS software and measured results are from SATIMO near-field antenna measurement system.

Fig. 22 shows a good agreement in comparison between the measured and the simulated reflection coefficients. It is seen that wide impedance bandwidth of $28 \%$ is realized that covers from 4.9 to $6.5 \mathrm{GHz}$.

In Fig. 23, it exhibits both the simulated and measured RHCP gains and AR bandwidths at the vertical plane $\varphi=225^{\circ}$ where the gain achieves its maximum as shown in Fig. 26. It is observed that the measured 3-dB AR bandwidth is achieved from 5.75 to $6.75 \mathrm{GHz}$, which can cover our objective uplink of standard C band: 5.85 to $6.425 \mathrm{GHz}$. In addition, the maximum RHCP gain of $4.9 \mathrm{dBic}$ is obtained within the AR bandwidth. For considering the omni-directional property, the realized AR bandwidth at the azimuth plane $\theta=32^{\circ}$ is presented in Fig. 24, which demonstrates the realized AR bandwidth is from 5.8 to $6.7 \mathrm{GHz}$ and the AR variations at 5.8, 6.25 , and $6.7 \mathrm{GHz}$ for all directions are small (Less than $1.5 \mathrm{~dB}$ )

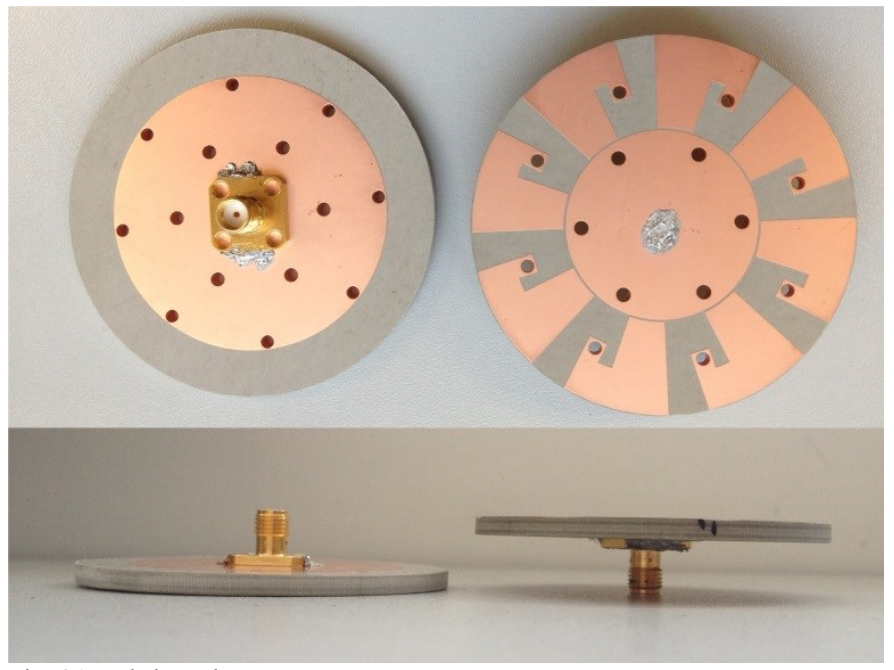

Fig. 21. Fabricated antenna.

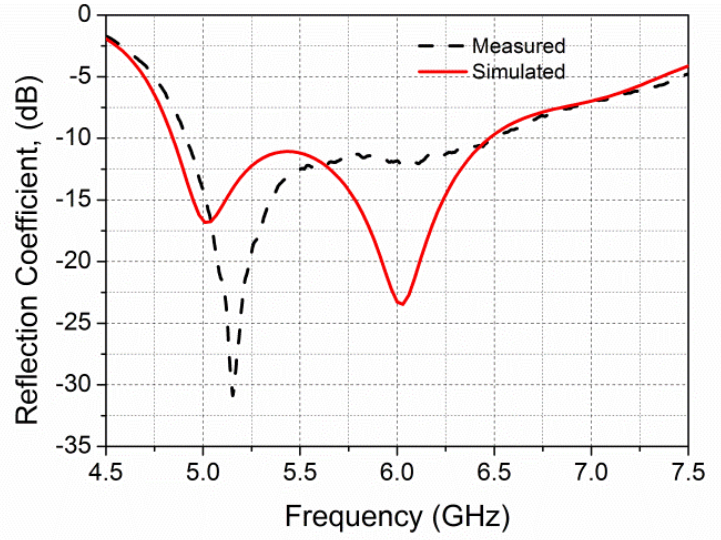

Fig. 22. Reflection coefficient of the proposed antenna

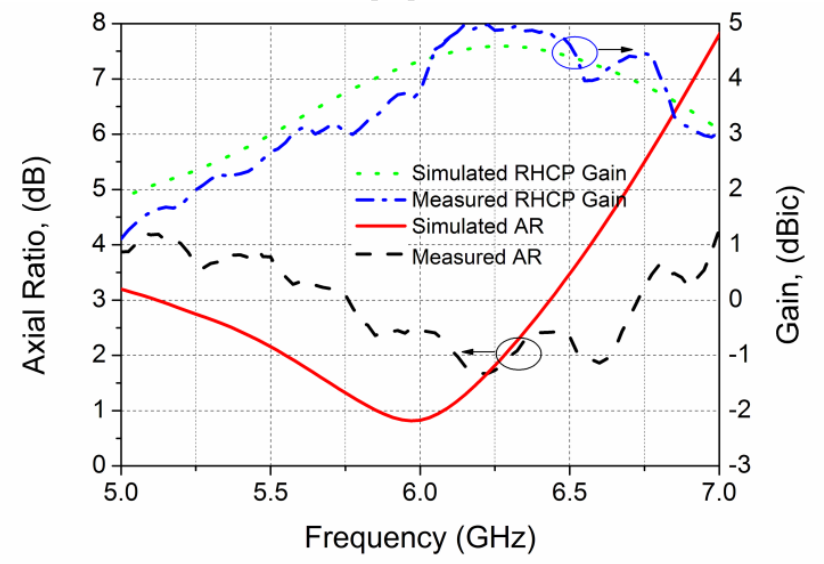

Fig. 23. RHCP gain and AR bandwidth at the vertical plane $\left(\theta=32^{\circ}\right)$.

The AR values at the vertical plane are also plotted in Fig. 25 which shows the AR beamwidth is wide. Within the required band, AR values are less than $3 \mathrm{~dB}$ when the angle $\theta$ sweeps from $15^{\circ}$ to $38^{\circ}$. Fig. 25 and Fig. 26 show the measured radiation patterns at the center frequency and the two band edge frequencies. This shows $\mathrm{CP}$ conical-beam radiation patterns have been obtained with the out of roundness less than $2 \mathrm{dBic}$ and the average RHCP gain is larger than $3.5 \mathrm{dBic}$. Maximum RHCP gain could reach 4.9 dBic.

Table I summarize and compare the performance of the different types of CP conical beam antennas. It is concluded 
TABLE I

Performance Comparison of CP CONicAl BeAm ANTENNAS

\begin{tabular}{lrrrrcc}
\hline \hline $\begin{array}{c}\text { Type of conical beam CP } \\
\text { antennas }\end{array}$ & $\begin{array}{c}\text { Center } \\
\text { frequency }\end{array}$ & $\begin{array}{c}\text { AR } \\
\text { bandwidth }\end{array}$ & Profile $(\mathrm{x} \times \mathrm{y} \times \mathrm{z})$ & Max. Gain & $\begin{array}{c}\text { Network } \\
\text { Required }\end{array}$ & $\begin{array}{c}\text { Fabrication } \\
\text { Complexity }\end{array}$ \\
\hline Patch [1] & $2.295 \mathrm{GHz}$ & $4 \%$ & $0.9 \lambda \times 0.9 \lambda \times 0.19 \lambda$ & $6.9 \mathrm{dBic}$ & Yes & Medium \\
Monopole [4] & $1.618 \mathrm{GHz}$ & $2.8 \%$ & $0.43 \lambda \times 0.43 \lambda \times 0.45 \lambda$ & $4.5 \mathrm{dBic}$ & Yes & $\begin{array}{c}\text { Medium } \\
\text { Simple }\end{array}$ \\
Dipole [3] & $4.5 \mathrm{GHz}$ & $3 \%$ & unknown $\times$ unknown $\times 0.47 \lambda$ & $0.6 \mathrm{dBic}$ & No & Sos \\
Loop [7] & $1.5 \mathrm{GHz}$ & $0.5 \%$ & unknown $\times$ unknown $\times 0.064 \lambda$ & $0.7 \mathrm{dBic}$ & Yes & Simple \\
Folded loop stubs [8-9] & $2.3385 \mathrm{GHz}$ & $<2 \%$ & $0.72 \lambda \times 0.72 \lambda \times 0.07 \lambda$ & $2.2 \mathrm{dBic}$ & No & Simple \\
Waveguide [5] & $12.5 \mathrm{GHz}$ & $4.8 \%$ & $3.2 \lambda \times 3.2 \lambda \times 1 \lambda$ & $7.5 \mathrm{dBic}$ & Yes & Complex \\
Monopole + Loop [10] & $4.025 \mathrm{GHz}$ & $0.5 \%$ & $0.56 \lambda \times 0.56 \lambda \times 0.09 \lambda$ & $4.5 \mathrm{dBic}$ & Yes & Complex \\
Monopole + Polarizer [15] & $5.3 \mathrm{GHz}$ & $54.9 \%$ & $0.9 \lambda \times 0.9 \lambda \times 0.43 \lambda$ & $6.3 \mathrm{dBic}$ & No & Complex \\
Patch + Feeding network [16] & $2.53 \mathrm{GHz}$ & $28.3 \%$ & $2.6 \lambda \times 2.6 \lambda \times 0.084 \lambda$ & $5.5 \mathrm{dBic}$ & Yes & Complex \\
Proposed Antenna & $6.25 \mathrm{GHz}$ & $14.4 \%$ & $1.16 \lambda \times 1.16 \lambda \times 0.06 \lambda$ & $4.9 \mathrm{dBic}$ & No & Simplest \\
\hline \hline
\end{tabular}

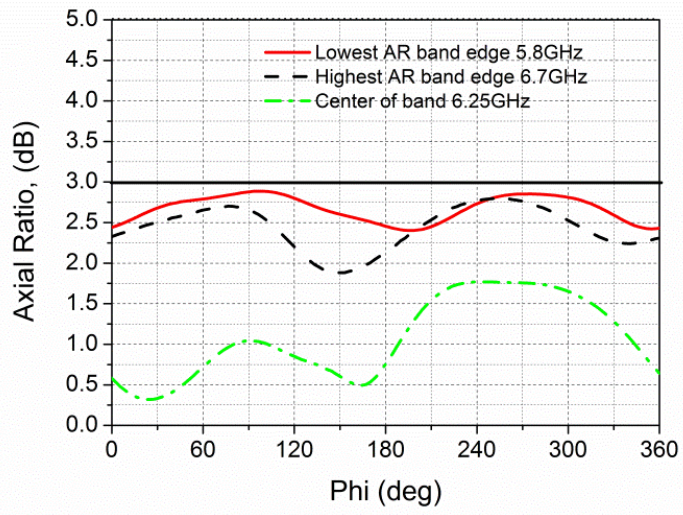

Fig. 24. Measured AR values at the azimuth plane $\left(\theta=32^{\circ}\right)$.

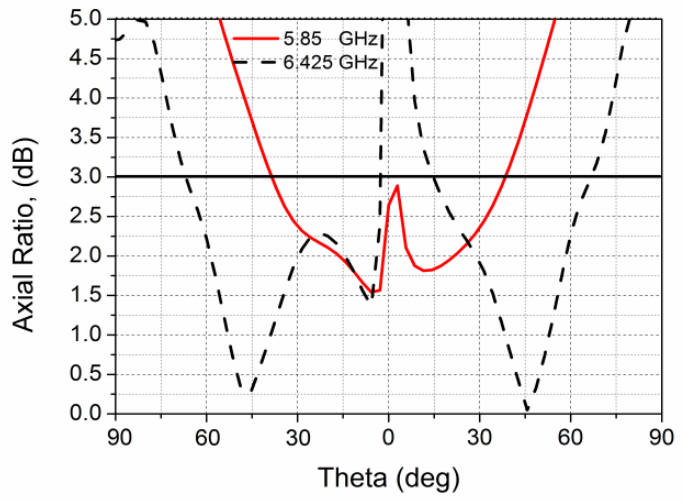

Fig. 25. Measured AR values of the vertical plane $\left(\varphi=0^{\circ}\right)$ at the two edge frequencies of uplink standard $\mathrm{C}$ band: $5.85 \mathrm{GHz}$ and $6.425 \mathrm{GHz}$.

that our proposed antenna is very low profile and exhibits wide impedance and AR bandwidths with good CP conical-beam radiation patterns. In addition, our design is simple which does not require additional feeding network.

It should be noted that the measured AR bandwidth at the vertical plane shifts to the higher frequency band. This is because ripples occur in the measured curve due to the uncertainties of alignment in the measurement system, such that the measured $\mathrm{AR}$ variation is more than $1 \mathrm{~dB}$ larger than the simulated result at the azimuth plane as seen in Fig. 17. Therefore, the AR bandwidth at the vertical plane will be affected accordingly. However, the problem of AR shifting can be solved by increasing the parameter $R_{\text {Loop }}$ in later fabrication process in order to shift it back to the design target since $R_{\text {Loop }}$ is sensitive to the AR bandwidth but impedance bandwidth as shown in Fig. 28.

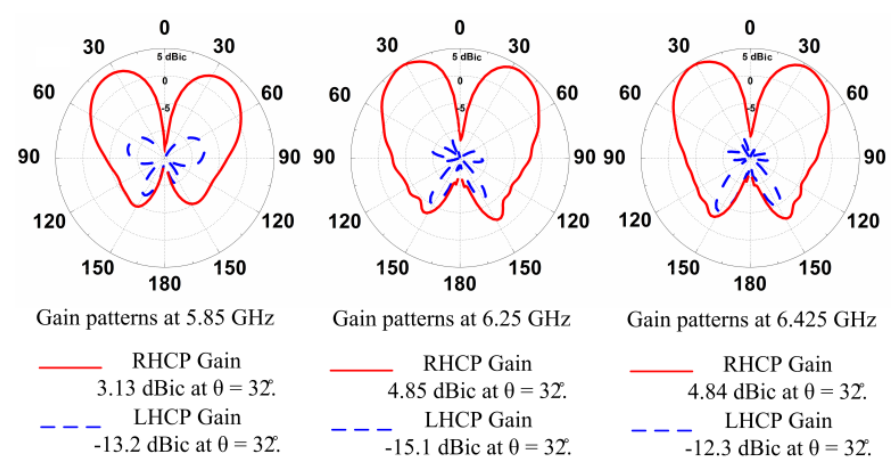

Fig. 26. Measured gain patterns at the vertical plane within uplink of standard C band.

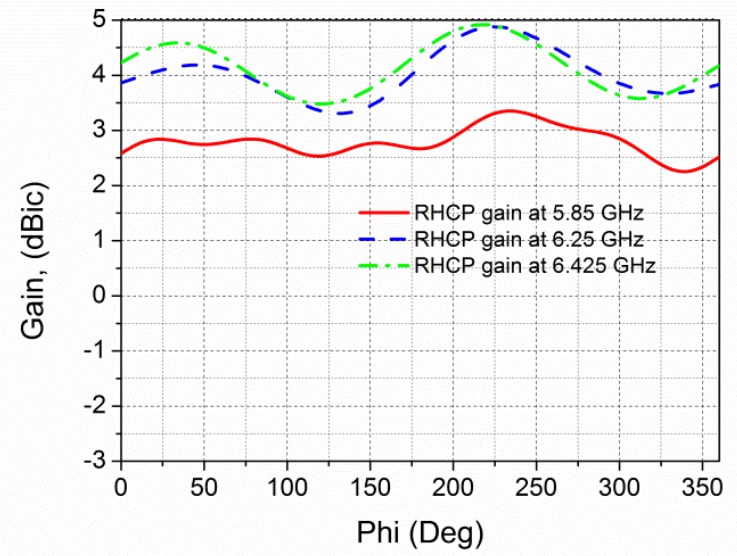

Fig. 27. Measured omni-directional gain at the azimuth plane $\theta=32^{\circ}$ within uplink of standard C band.

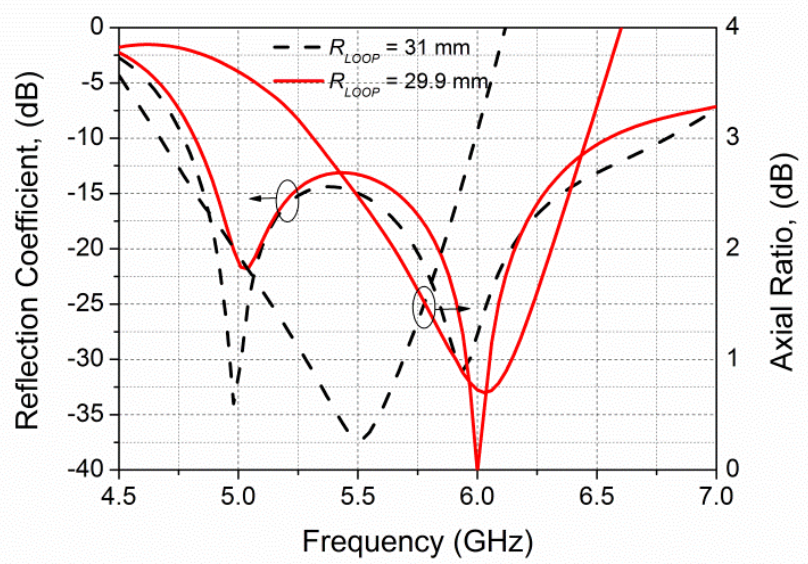

Fig. 28. Impedance and AR bandwidths variation by changing the parameter $R_{\text {Loop. }}$ 


\section{CONCLUSION}

A circularly-polarized conical-beam antenna with low profile and wide bandwidth has been proposed. A marriage of the center-fed monopolar patch and the coupling loop stubs has been introduced to achieve the wideband $\mathrm{CP}$ radiation. The operation principle and key considerations in the antenna design have been discussed. A prototype working at the standard $\mathrm{C}$ band has been fabricated and tested. The measured results are in a good agreement with the simulations, which shows wide impedance and AR bandwidths, stable gain and the excellent omni-directional characteristics. It should be a good candidate for the satellite communications.

\section{ACKNOWLEDGMENT}

The authors wish to acknowledge Miss. Xia Gao and Dr. Ko kan So for their help in antenna fabrication. They would also like to express their gratitude to Prof. Chi hou Chan and Dr. Hau wah Lai for their advance and Mr. Kai xu Wang for help in radiation pattern measurement.

\section{REFERENCES}

[1] J. Huang, "Circularly polarized conical patterns from circular microstrip antenna," IEEE Trans. Antennas Propag., vol. 32, no.9, pp. 991-994, Sep. 1984.

[2] W. Liu, K. Wei, Z. Zhang, J. f. Zheng, and Z. h. Feng, "A circularly polarized antenna with conical beam," IEEE Symposium on Electrical Design of Advanced Packaging and Systems (EDAPS), 2011

[3] A. NeSid, V. Brankovid and I. Radnovid, "Circularly polarised printed antenna with conical beam," Electronics Letters, vol. 34, no. 12, pp. 1165-1167, June. 1998.

[4] H. Kawakami, G. Sato and R. Wakabayashi, "Research on circularly polarized conical-beam antennas," IEEE Antennas and Propagation Magazine, vol. 39, no. 3, pp. 27-39, June. 1997.

[5] S. S. Qi, W. Wu, and D. G. Fang, "Singly-fed circularly polarized circular aperture antenna with conical beam," IEEE Trans. Antennas Propag., vol. 61, no.6, pp. 3345-3349, June. 2013.

[6] J. S. Row and M. C. Chan," Reconfigurable circularly-polarized patch antenna with conical beam," IEEE Trans. Antennas Propag., vol. 58, no.8, pp. 2753-2757, Aug. 2010

[7] H. Nakano, K. Fujimori, and J. Yamauchi, "A low-profile conical beam loop antenna with an electromagnetically coupled feed system," IEEE Trans. Antennas Propag., vol. 48, no.12, pp. 1864-1866, Dec. 2000.

[8] K. Phaebua, C. Phongcharoenpanich, D. Torrungrueng, N. Surittikul, and W. Villarroel, "An eight-branch folded strip antenna with a circular parasitic patch on circular ground plane for SDARS application," IEEE Antennas and Propagation Society International Symposium, 2009.

[9] R. Suwalak, C. Phongcharoenpanich, D. Torrungrueng, N. Surittikul and W. Villarroel, "A probe-fed eight-branch folded strip with notched-annular-ring parasitic patch antenna on circular ground plane," International Symposium on Antennas and Propagation, 2009.

[10] W. D. Liu, K. P. Wei, Z. J. Zhang, J. F. Zheng, and Z. H. Feng, "A circularly polarized antenna with conical beam," Electrical Design of Advanced Packaging and Systems Symposium (EDAPS), 2011.

[11] J. Takada, A. Tanisho, K. Ito, and M. Ando, "Circularly polarised conical beam radial line slot antenna," Electronics Letters, vol. 30, no. 21, pp. 1729-1730, Oct. 1994.

[12] K. Ito, J.P. Daniel and J. Lenormand, "A printed antenna composed of strip dipoles and slots generating circularly polarized conical patterns," International Symposium on Antennas and Propagation Society, 1989.

[13] C. L. Deng, P. H. Li and W. Q. Cao, "Low-profile patch antennas with vertical monopole-Like radiation patterns based on modified capacitive coupling structures," Antennas and Wireless Propagation Letters, vol. 11, pp. 1354-1357, 2012

[14] H. Nakano, H. Oyanagi and J. Yamauchi, "A wideband circularly polarized conical beam from a two-arm spiral antenna excited in phase," IEEE Trans. Antennas Propag., vol. 59, no.10, pp. 3518-3525, Oct. 2011.
[15] Y. M. Pan and K. W. Leung, "Wideband circularly polarized dielectric bird-nest antenna with conical radiation pattern," IEEE Trans. Antennas Propag., vol. 61, no.2, pp. 563-570, Feb. 2013.

[16] K. L. Lau and K. M. Luk, "A wideband circularly polarized conical beam patch antenna," IEEE Trans. Antennas Propag., vol. 54, pp.1591-1594, May 2006.

[17] J. Liu, Q. Xue, H. Wong, H. W. Lai, and Y. L. Long, "Design and analysis of a low-profile and broadband microstrip monopolar patch antenna," IEEE Trans. Antennas Propag., vol. 61, no.1, pp.11-18, Feb. 2013.

[18] K. P. Wei, Z. J. Zhang and Z. H. Feng, "Design of a wideband horizontally polarized omnidirectional printed loop antenna," Antennas and Wireless Propagation Letters, vol. 11, pp. 49-52, 2012.

[19] L. Bras, N. Borges Carvalho and P. Pinho, "Omnidirectional printed loop antenna for taxi communications," Antennas and Propagation Society International Symposium (APSURSI), 2013.

[20] H. Nakano, R. Satake and J. Yamauchi, "Horizontally polarized, omnidirectional antenna with a single feed," IEEE International Conference on Wireless Information Technology and Systems (ICWITS), 2010 .

Wei Lin was born in Lanzhou, Gansu Province, China. He received the B.E. degree in information engineering and the M.E. degree in electromagnetic field and microwave engineering from South China University and Technology, Guangzhou, China, in 2009 and 2012, respectively. He was working as research associate in Nanyang Technological University, Singapore from August 2012 to July 2013. He is currently pursuing his $\mathrm{PhD}$ degree at City University of Hong Kong, Hong Kong S. A. R., China. His research interests include antennas, RF circuit and RFIC, etc.

Hang WONG received the B.Eng., M.Phil., and Ph.D. degrees in electronic engineering from City University of Hong Kong in 1999, 2002 and 2006, respectively. He joined the State Key Laboratory (SKL) of Millimeter Waves, in Hong Kong SAR, China, in 2008 as a senior engineer. He was an acting assistant professor in the Department of Electrical Engineering, Stanford University, in 2011. He joined the Department of Electronic Engineering as an assistant professor at City University of Hong Kong in 2012. His research interests include design of broadband antennas, small antennas, GPS antennas, millimeter wave antennas, and terahertz devices and applications. He has published more than 70 international journals and conference papers. He was awarded 14 US and China patents. He was the co-author of two antenna research book chapters. He is the deputy director of Information Communication Technology Centre of City University of Hong Kong in Shenzhen. He is the chair of the IEEE Hong Kong Section of the Antennas and Propagation (AP)/Microwave Theory and Techniques (MTT) joint chapter. Dr. Wong was awarded the Outstanding Research Thesis Award from City University of Hong Kong in 2002. He received the Microwave Student Prize at the Asia Pacific Microwave Conference 2006 held in Yokohama, Japan; and received the Best Paper Award at the International Symposium on Antennas and Propagation 2008 in Taipei. He received 2011 State Technology Invention Award presented by the Ministry of Science and Technology of the P.R. China 